\title{
An Overview on Wafer Pre-Aligner
}

\author{
Xianwen Zhuang ${ }^{1, \mathrm{a}}$, Peng Zhang ${ }^{1, \mathrm{~b}}$, Lina Hao ${ }^{1, \mathrm{c}}$, Chengbao Huang ${ }^{1, \mathrm{~d}}$ \\ ${ }^{1}$ School of Mechanical Engineering and Automation, Northeastern University, China \\ a wonderdsr@163.com, bz.pyj@163.com, cneuhaolina@163.com, dbbsj2006@126.com
}

Keywords: Wafer pre-alignment, Structure of pre-aligner, Machinery vision, Circle fitting algorithm

\begin{abstract}
Wafer pre-aligner plays an important role in wafer manufacture. Wafer pre-aligner positions wafers accurately before they are transferred onto exposure platform, and the positioning accuracy greatly affects the exposure accuracy of wafer and the work efficiency of the whole manufacture system. The paper makes an overview on wafer pre-aligner from the following aspects: working principle, mechanical structure, machine vision and the algorithm on the detection of wafer edge.
\end{abstract}

\section{Introduction}

Wafer pre-aligner is an apparatus used to precisely position the silicon wafers before photo-etching. Because the accuracy requirement of machining is higher than that of wafer transportation, we need to position wafers with wafer pre-aligner to compensate the position error produced during transportation. As the improvement of manufacturing process of wafer, it makes demands of higher accuracy and quicker alignment speed of wafer pre-aligner system. Take the IPA-300VS-1 wafer pre-aligner ${ }^{[1]}$ system produced by ISEL-ROBOTIK as an example, its position accuracy of notch can reach $\pm 0.05^{\circ}$ and position accuracy of center can reach $\pm 0.0025 \mathrm{~mm}$. Its position time is $3.5 \mathrm{~s}$.

The pre-aligners can be classified into two types, the mechanical and the optical. The mechanical type usually uses mechanical structure to position the center of wafers, and its position accuracy is too low to satisfy the high accuracy requirement today. The optical type is widely used in wafer pre-alignment with its high position accuracy today.

The optical type uses optical sensor and mechanical structure to realize high accuracy position of wafer. A typical optical wafer pre-aligner is shown in fig. $1^{[2]}$. The alignment process is as follows: Firstly, rotation unit rotates wafers for one round or more. Then the optical sensor unit detects the edge information of wafer and calculates out the central deviation and notch position, and sends the results to controller, which then sends out signals to the mechanical structure. According to the results, the rotation unit rotates wafer to make the center deviation line in the $\mathrm{Y}$ direction. The centering unit moves wafer in a straight line along $\mathrm{Y}$ direction to finish the centering process. Finally, the rotation unit rotates wafer and locates the notch at a specified position.

\section{Mechanical Structure}

As shown in fig. 1, the mechanical structure can be divided into two parts: gripping mechanism and actuating mechanism, as shown in fig. 1.

Gripping Mechanism. The wafer gripping mechanism can generally be classified into two types: vacuum chuck and edge clamping mechanism. 
Vacuum chuck is widely used in wafer gripping, which can only works in atmosphere environment. Using vacuum chuck to hold wafer can well protect wafer from being damaged. As shown in fig. $2^{[3]}$, Part 26 is a vacuum chuck and part 28 is its upper part which contacts wafer. Part 40 is a vacuum pump, with which can produce or release pressure differences on wafer to hold or let off wafer.

There are some disadvantages of vacuum chuck. Its chuck directly contacts the back of wafer, which contaminates wafer to a certain degree. Besides, it doesn't work in vacuum environment.
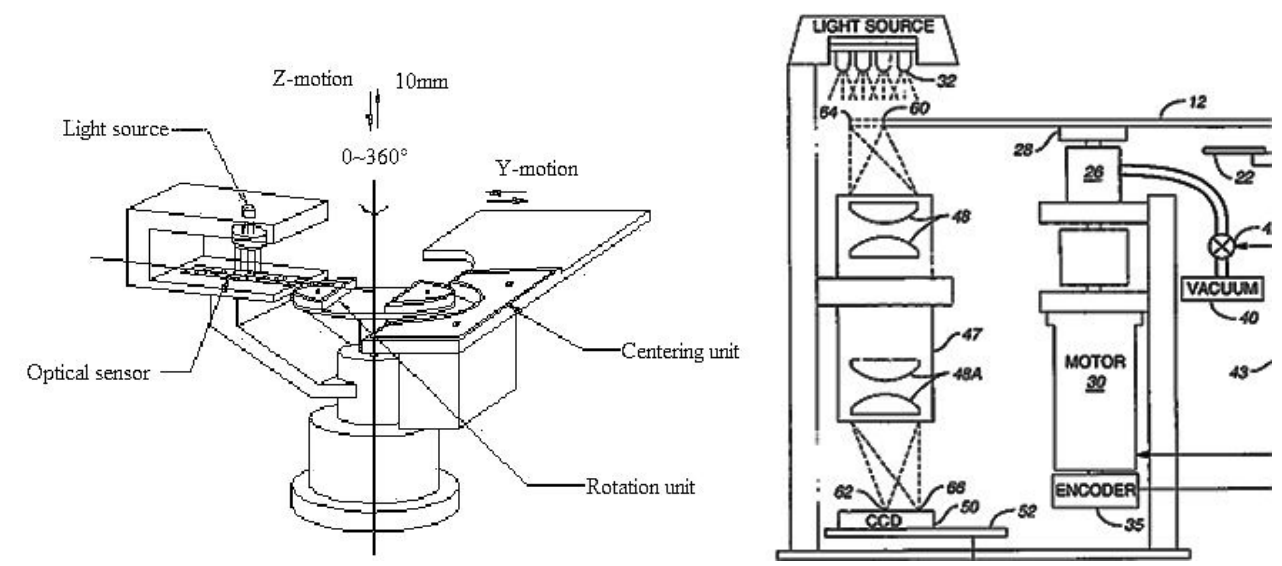

Fig. 1. An illustration of optical wafer pre-aligner

Fig. 2. Wafer pre-aligner with vacuum chuck and machine vision system

Edge clamping is another method to hold wafer. It clamps on the edge of wafer, which prevents the contamination at the back of wafer and can work in vacuum environment. An edge clamping mechanism is shown in fig. $3^{[4]}$. It turns the up-and-down motion to side-to-side movement with a linkage 13, which then results the release and clamping motion.

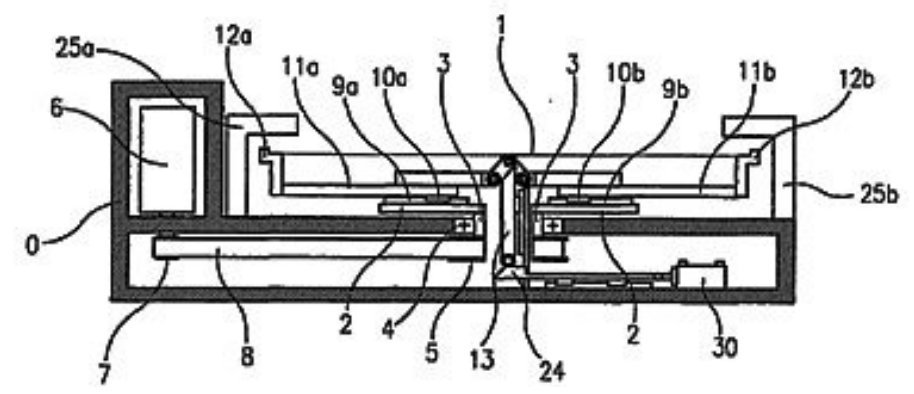

Fig. 3. Edge clamping mechanism

Actuating Mechanism. Actuating mechanism of a wafer pre-aligner consists of three units: rotation unit, centering unit and elevating unit.

Rotation unit is to rotate wafer about $\mathrm{Z}$ axis. Rotation unit works in three processes. The first process is the detection of wafer edge information with optical sensor, in which rotation unit drives wafer to rotate about $\mathrm{Z}$ axis for one round or more to collect enough wafer edge information. The second process is rotating wafer to make the center deviation line in the $\mathrm{Y}$ direction. The third process is to rotate wafer and locate the notch at a specified position.

Centering unit is used to make the rotation center coincide with wafer center. When the center deviation has been rotate to $\mathrm{Y}$ direction, centering unit performs a linear Y-motion to make the wafer center coincide with rotation center.

Elevating unit generally plays a part in wafer handover between rotation unit and centering unit.

\section{Machine Vision System}

Machine vision system consists of optical sensor, light source and optical lens, as shown in fig. 2. Part 32 is the light source, part 48 is a group of optical lens and part 50 is the CCD optical sensor. 
Optical Sensor. Charged Couple Device (CCD) is mostly used as the optical sensor in wafer pre-aligner. It detects the edge information of wafer and calculates out the center and notch position of wafer, the algorithm of which is not complex. Another optical sensor is CCD camera, which takes pictures of wafer and calculates out the position information according to image process algorithm. The algorithm is complex, but it can deal with wafers whose radius is larger than $300 \mathrm{~mm}$ well.

Light Source. There lies high requirement of detection speed and accuracy, and also the wafer is always in motion, so a linear light source ${ }^{[10]}$ which owns short light integration time and satisfies the high requirement of detection speed and accuracy is needed.

Optical Lens. The beams transmitted by a linear light source are astigmatic elliptical beams. It needs optical lens to collimate the beams in order to gain parallel beams ${ }^{[11,13]}$. Theoretical research suggests that it should adopt aspheric lens to meet high requirement of collimation ${ }^{[1]}$. Hyperboloid lens are the easiest to be fabricated among aspheric lens and are used widely.

\section{Algorithms of Circle Fitting}

There are many algorithms of circle fitting, such as mass point calculation method ${ }^{[12,14]}$, weighted average method ${ }^{[6]}$, circle periphery fitting method, least square method, Hough transform ${ }^{[8,9]}$, et al. The weighted average method has a simple algorithm and fast processing speed, but the fitting effect is not so well. The fitting results of circle periphery fitting and least square method are much better than weighted average method. Besides, the processing speed of these two methods is acceptable. The fitting effect of Hough transform is also well but the algorithm is too complex which reduces its processing speed.

Circle Periphery Fitting. The mathematical model ${ }^{[2,5]}$ is shown in fig. 4.
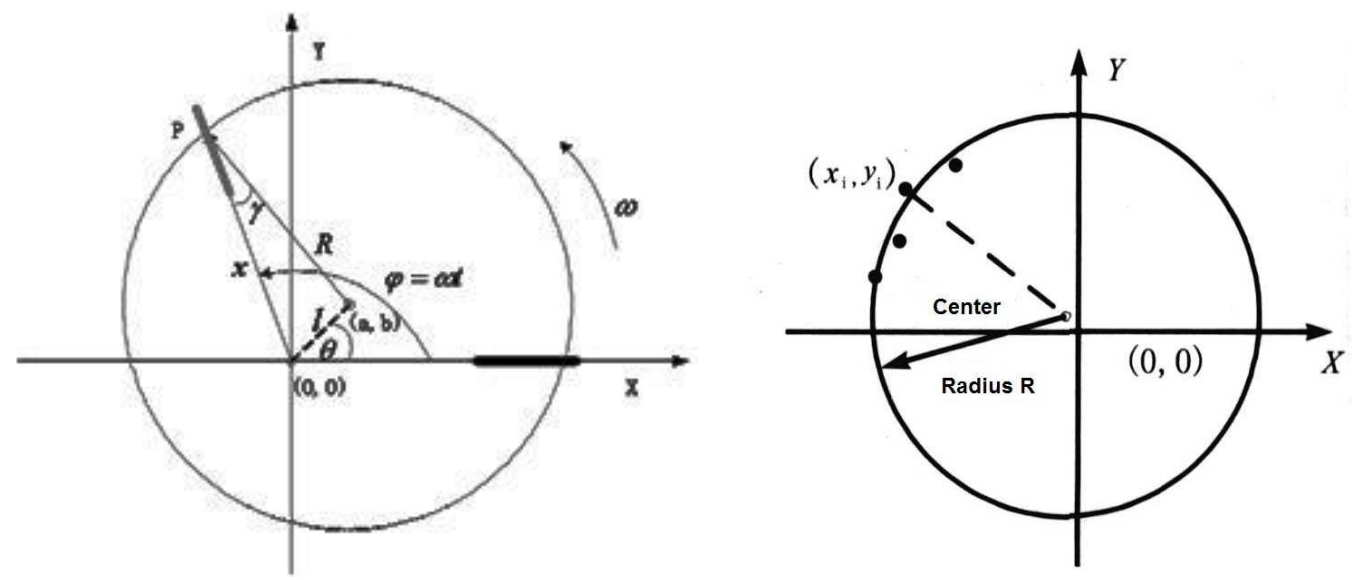

Fig. 4. Circle periphery fitting

Fig. 5. Least square circle fitting method

Through analysis we can get the relationship between the sample data $\mathrm{x}, \varphi$ and radius of wafer $\mathrm{R}$, position parameters $l$ and $\theta$ :

$$
\mathrm{x}=l \cos (\varphi-\theta)+\sqrt{R^{2}-l^{2} \sin ^{2}(\varphi-\theta)}
$$

Take $l, \mathrm{R}, \theta$ as the parameters to be fitted and construct the function as follows:

$$
\mathrm{S}=\sum_{i=1}^{N}\left[x_{i}-\left(l \cos \left(\theta+\varphi_{i}\right)+\sqrt{R^{2}-l^{2} \sin ^{2}\left(\theta+\varphi_{i}\right)}\right)\right]^{2}
$$

$S$ is the squares sum of the distances between measuring point and fitted circle. The fitted circle which minimizes $\mathrm{S}$ is the best fitted circle.

Least square method. The least square method ${ }^{[6,7]}$ is to get a function $\psi(x)$ which fits sample data $\left(x_{i}, y_{i}\right)$ and makes the square sum of the difference between $\psi\left(\mathrm{x}_{i}\right)$ and $y_{i}$ minimum. 
The circle function can be written as $x^{2}+y^{2}+a x+b y+c=0$. According to the method proposed by Kasa ${ }^{[2]}$, we let denote $Q(\mathrm{a}, \mathrm{b}, \mathrm{c})=\sum \delta_{i}{ }^{2}$, where $\delta_{i}=\mathrm{d}_{i}{ }^{2}-\mathrm{R}^{2}$ and $\mathrm{d}_{i}$ is the difference of the distance between sample point and circle center and circle radius $\mathrm{R}$, as shown in fig. $5^{[5]}$. Make the partial differentiation of the function equals zero, we can get three equations and then solve out the value of $a, b, c$ and calculate out the parameters of circle finally.

\section{Algorithms of Notch Detection}

Notch detection ${ }^{[2,10]}$ is performed in two stages. One is the stage to get wafer circle information. It needs to discard the notch data to fit the circle more precisely by notch detection. The other is the stage to locate the notch position after having finished wafer centering. It needs to detect notch position information so as to turn the notch to a specified direction.

The edge rate of notch is very different with the other area of wafer, so we can set a threshold to distinguish the notch area from the other area of wafer. We can set the center of notch area as the reference point. For the notch is a circular arc, we can gain information through circle fitting algorithm mentioned above.

\section{Conclusion}

Wafer pre-aligner is an important apparatus in wafer fabrication system. Here we make an overview on wafer pre-aligner after consulting many materials about wafer pre-aligner. We hope it can help people make quick understand of wafer pre-aligner.

\section{Acknowledge}

The authors gratefully acknowledge that the work was supported by the 985 Project of Northeastern University of China and fully appreciate the guidance and help of teachers and seniors.

\section{References}

[1] Du Yu, The Development of Wafer Pre-aligner, Dalian University of Technology, 2006.

[2] Li Chunjiang, Research on Wafer Pre-Alignment System, Harbin Institute of Technology, 2006.

[3] Sagues et al, Wafer Aligner System, U.S. Patent 6275742B1 (2001).

[4] Keisuke Yoshino et al, Aligner, U.S. Patent 20090053029A1 (2009).

[5] Rong Weibin et al, Research on Wafer Pre-aligner System, Robot, 2007, 29(4): 331-336.

[6] Xu Guowang, Liao Mingchao, Methods of Circle Fitting, J. Journal of Wuhan Polytechnic University, 2002, 21(4):104-106.

[7] Umbach Dale, Jones Kerry N, A few methods for fitting circles to data, IEEE Trans on Instrum Meas, 2003, 52(6):1881-1885.

[8] Li Shuhua, Tie Yong, A Robust High-Precision Circular Target Detection Method Based on Hough Transform, ICCASM , 2010(14):253-257.

[9] A. Goneid, A Method For The Hough Transform Detection of Circles and Ellipses Using a 1-Dimensional Array, IEEE International Conference on Systems, Man, and Cybernetics, 1997:3154-3157.

[10]Kong Xiangji, The Research of Vision System Based on Wafer Pre-aligner Robot, Dalian University of Technology, 2007.

[11]Hee-Sub Lee et al, A 12-inch wafer prealigner, Microprocessors and Microsystems, 2003(27): $151-158$.

[12]Z. Fu et al, Wafer prealigning robot based on shape center calculation, Industrial Robot, 2008, 35(6):536-540.

[13]Hee Sub Lee et al, The Development of a 12-inch Wafer Prealigner with Optical Character Recognition, 27th Annual Conference of the IEEE Industrial Electronics Society, 215-219.

[14]Chunxia Huang et al, The development of a wafer prealigner based on the multi-sensor integration, Assembly Automation, 2008,28(1): 77-82. 